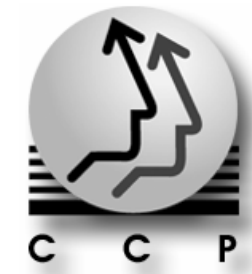

Población y Salud en Mesoamérica

Revista electrónica publicada por el

Centro Centroamericano de Población,

Universidad de Costa Rica, 2060 San José, Costa Rica

http://ccp.ucr.ac.cr

Población y Salud en Mesoamérica

Revista electrónica semestral, ISSN-1659-0201

Volumen 8, número 2, artículo 3

Enero - junio, 2011

Publicado 1 de enero, 2011

http://ccp.ucr.ac.cr/revista/

\title{
La lógica de lo adecuado en materia de saneamiento ambiental en asentamientos humanos en Costa Rica
}

\author{
Horacio Alejandro Chamizo Garcia
}

\author{
(cc) EY-NC-ND \\ Protegido bajo licencia Creative Commons \\ Centro Centroamericano de Población
}




\title{
La lógica de lo adecuado en materia de saneamiento ambiental en asentamientos humanos en Costa Rica
}

\section{The logic of what is appropriate in terms of environmental sanitation in human settlements in Costa Rica}

\author{
Horacio Alejandro Chamizo García ${ }^{1}$
}

\section{RESUMEN}

Se desarrolla un análisis de contenido del marco normativo formal del saneamiento ambiental en asentamientos humanos en Costa Rica. El punto de partida teórico es el nuevo institucionalismo y la teoría de redes de políticas públicas que orientó la recopilación de leyes y reglamentos en cuatro escenarios de gestión que deben funcionar articuladamente. El análisis de contenido evidenció que la desarticulación de las reglas del juego y la concentración del poder en los técnicos no favorece el abordaje del problema en su complejidad así como la gestión en red y la construcción de una ciudadanía activa que permita poner en práctica el principio enunciado de la producción social de la salud.

Palabras clave: saneamiento ambiental, gestión de políticas públicas, neoinstitucionalismo

\begin{abstract}
Develops a content's analysis of the formal normative framework of the environmental sanitation in human settlements in Costa Rica. The theoretical point of departure is the new institutionalisms and the theory of networks of public policy that oriented the selection of laws and regulations in four settings of management that should function articulated. The analysis of content showed that the dismemberment of the play's rule and the concentration of the power in the technicians, does not favor the problem's approach in its complexity, the network management and the construction of an active citizenship that permit to put in practice the principle statement of the social production of the health.
\end{abstract}

Keyword: environmental sanitation, management of public policy, new institutionalism

Recibido: 13 jul. 2010

Aprobado: 18 oct. 2010

\footnotetext{
${ }^{1}$ Profesor e investigador de la Escuela de Tecnologías en Salud de la Facultad de Medicina de la Universidad de Costa Rica. horacio.chamizo@ucr.ac.cr. COSTA RICA
} 


\section{LA RELEVANCIA DEL MARCO NORMATIVO FORMAL}

El reconocimiento internacional de que los problemas de saneamiento ambiental se encuentran irresueltos para una buena parte de la humanidad, ha significado una mirada crítica a pesar de los logros, sobre la Década Internacional del Agua Potable y el Saneamiento. En febrero del año 2000 se llevó a cabo una reunión de expertos en Bellagio, Italia, en la que se propusieron principios orientadores como fundamento para el desarrollo de servicios de saneamiento ambiental más efectivos. Se definieron así los Principios de Bellagio, adoptados por agencias internacionales como el Consejo Coordinador para el Suministro de Agua y Saneamiento (WSSCC), durante su Quinto Foro Mundial en Iguazú, Brasil, en noviembre del 2000.

Los principios de Bellagio buscan llamar la atención sobre uno de los satisfactores más importantes de las condiciones de vida de la población y que explican de una manera fuerte, la pobreza y las condiciones ambientales que determinan la salud de la población en general. Los servicios de saneamiento ambiental insuficientemente ofrecidos en muchos países, pueden ser replanteados para que sean sostenibles ambientalmente y para que respondan directamente a las expectativas de la población beneficiaria, si se transforma el modelo de gestión propiciando la construcción de una ciudadanía activa. (Insituto Federal Suizo de Ciencia y Tecnología Acuática, 2006)

El contexto sociopolítico de la gestión del saneamiento ambiental se ha estado transformando con los procesos de reforma del Estado. Sin embargo, existe desatención en ciertos sectores de la población y un incremento de la brecha socioeconómica y la inequidad. A propósito de los problemas de saneamiento ambiental y de las deficiencias en cobertura y calidad de estos servicios, se reconoce que existe un rezago en gobernabilidad que actúa como un verdadero obstáculo para el desarrollo. (OMS, Comission on Social Determinans of Health, 2007)

La Comisión sobre Determinantes Sociales de la Salud (2007) que organiza la Organización Mundial de la Salud, reconoce el papel que tiene el escenario geográfico y la Sociedad Civil, por lo que le asigna un rol relevante a la incorporación de actores. Este rol tiene que ver con la identificación de esos determinantes (particularmente los ambientales) y establecer relaciones entre actores para construir socialmente el conocimiento y las acciones. Se le asigna un valor trascendental para la gestión local de la salud. (OMS, Comission on Social Determinans of Health, 2007)

Un elemento fundamental en las transformaciones propuestas es que el saneamiento ambiental no es solo un espacio de acción técnico, sino que también es social y político. Sin embargo, el modelo de gestión pública burocrático y posteriormente, el gerencialista, han establecido un marco normativo que no favorece necesariamente la gobernanza democrática. Esto significa que es poco lo que se puede avanzar en la dirección del cambio sin revisar y transformar el marco institucional que sustenta la gestión.

La presente investigación se propone revisar el marco normativo formal del saneamiento ambiental a nivel de asentamientos en Costa Rica, para de esta forma identificar problemas de diseño institucional que pueden afectar la gestión de las políticas públicas. A continuación se 
explican los principales supuestos teóricos y metodológicos que sustentan su pertinencia y validez.

\section{LAS NORMAS FORMALES Y EL COMPORTAMIENTO DE LOS ACTORES EN LA GESTIÓN: FUNDAMENTOS TEÓRICOS Y METODOLÓGICOS}

La presente investigación parte del supuesto de que el ser humano posee cualidades limitadas para formular y resolver problemas complejos en comparación con la amplitud de los problemas que enfrentan las políticas públicas. Se presupone que los individuos no se comportan y toman decisiones absolutamente racionales en función de sus intereses personales y buscando optimizar sus beneficios como lo plantea el enfoque de análisis de políticas públicas pluralista-racionalista, sino se limitan bajo "reglas del juego" (instituciones).

Para estudiar la relación entre el Estado y la Sociedad, establecida a través de las políticas públicas, se ha desarrollado como uno de los modelos teóricos el nuevo institucionalismo, situado en una posición intermedia entre las teorías pluralistas que suponen el racionalismo economicista y por otro lado el supuesto de la supeditación de la sociedad a un Estado absolutamente parcial y tomado por una minoría empoderada económicamente. (Giandoménico, 1998).

Las teorías pluralistas como la Teoría de Juegos, aplicada en el análisis de políticas públicas, se enfoca en un individuos racional, un actor inteligente y dotado de información para la toma de decisiones, de una racionalidad absoluta. Este actor es capaz de tomar decisiones conscientes cual cliente en el mercado.

Por otra parte, las teorías que le conceden un papel más trascendente al Estado, se apartan de la idea de la racionalidad absoluta. Lejos de la idea de la racionalidad absoluta, no suponen un comportamiento consciente de los actores, se instintivo, estructurado por el Estado. En el caso del neomarxismo, el Estado no es neutral políticamente, está tomado por los intereses particulares de los dueños de los medios de producción.

Como parte del nuevo institucionalismo se le concede una notable importancia a la estructura del Estado con sus instituciones, también a las reglas y procedimientos así como a las organizaciones que se integran al sistema político. Frente al institucionalismo clásico con carácter formal y legal se presenta un nuevo enfoque funcional y descriptivo que revaloriza a las instituciones (Rhodes, 1997), otorgando relevancia a los actores.

Bajo el enfoque neoinstitucionalista no solo las instituciones tienen un papel preponderante, sino el Estado y las organizaciones. Se le asigna un rol muy importante a los procesos de socialización y participación (Rivas Leone, 2003). En el enfoque neoinstitucionalista, las instituciones proporcionan el marco de valores y normas dentro de los cuales se establece la conducta humana. Para Douglas North (1993), las instituciones son las reglas del juego en una sociedad o son las limitaciones ideadas por el ser humano que dan forma a la interacción entre actores. (North, Instituciones, cambio social y desempeño económico, 1993). 
Existen instituciones formales e informales. Las formales se refieren al marco normativo que determinan las leyes, reglamentos, contratos; las informales se refieren a códigos de conducta humana como valores, normas sociales de comportamiento, percepciones, elementos culturales en general. Este marco normativo constriñe, limita el comportamiento de los actores.

Las instituciones se forman a partir de la estructura de significaciones, rasgo que concuerda en cierta medida con lo que se asume en las vertientes sociológicas e históricas del neoinstitucionalismo. Estas significaciones no son entonces sucesos espontáneos sino procesos más o menos largos de inserción de los actores en un contexto. La distinción de la perspectiva normativa respecto a la sociológica es que esa estructura de significados termina configurando las normas formales que a su vez determinan la lógica de lo que es adecuado hacer. La estructura de normas formales e informales se retroalimentan históricamente reforzándose o debilitándose. (Peters, 2003)

Para Rangeon (1986) las normas se perciben como modelos de comportamiento que señalan la lógica de lo que se debe hacer. Sin embargo, es un proceso flexible en la medida que se transforma la sociedad y cambia la percepción de lo que se debe hacer. En esta media gozan de legitimidad porque representan el interés general (Rangeon, 1986). Se entiende como un proceso de legitimación, normalización de comportamientos y unificación de reglas y se puede interpretar como un sistema coherente de representaciones sociales mencionado más arriba cuando se explicó el neoinstitucionalismo sociológico.

El diseño institucional queda así configurado como una plantilla o modelo prescriptivo del comportamiento de los actores. Sin embargo, los individuos tienen la capacidad de escoger las influencias y deben interpretar los compromisos institucionales, de manera tal que se asigna cierta capacidad racional. Se señala al menos un punto de contacto con la lógica de los modelos más racionalistas en el momento de definir lo que es adecuado, en la medida que se espera que los individuos piensen en primer lugar en el beneficio concreto que obtienen como consecuencia de sus actos. (Peters, 2003)

Una vez explicado el punto de partida teórico relativo al análisis de políticas públicas, es conveniente definir conceptos de la arena en cuestión: el saneamiento ambiental. El concepto de saneamiento ambiental considerada en la presente investigación se distancia de la definición tradicional. El saneamiento ambiental se define tradicionalmente como conjunto de servicios: aguas residuales, pluviales, residuos sólidos, y agua para consumo humano (Insituto Federal Suizo de Ciencia y Tecnología Acuática, 2006). Pero esta definición contiene un claro sesgo hacia el modelo de gestión dominante (el burocrático en alternancia con el gerencialista), y por lo tanto, tiene implicaciones para los habitantes de los asentamientos.

Desde el punto de vista sociopolítico, la definición tradicional de saneamiento ambiental supone que los habitantes de los asentamientos son usuarios de servicios, concebidos de forma fragmenta por distintos proveedores que operan en condiciones muy cercanas a las del mercado, sujeto a una propuesta técnica definida desde "arriba" por gerentes o administradores públicos que priorizan objetivos de eficiencia y calidad. Estos objetivos no necesariamente se ajustan a las expectativas de desarrollo local sostenible y probablemente terminan dejando insatisfechas las necesidades de los habitantes quienes reciben servicios insuficientes, promoviendo así la inequidad ya que se enfocan hacia la eficiencia y la contención de costos. 
La gestión del saneamiento ambiental debe enfrentar entonces la importante la ruptura de paradigmas tradicionales sobre el proceso salud-enfermedad que ha promovido la administración pública asumiendo posiciones biomédicas por una parte y tecnocráticas por otra. Este desafío se produce en el marco de los procesos de reforma del Estado. Se produce en un ambiente político con implicaciones para la adopción de principios que buscan potenciar la eficiencia de la gestión pública en una situación de mayor escasez de recursos para la implementación de políticas (Sojo, 2000). Así mismo existe la necesidad de involucrar a la Sociedad Civil en la toma de decisiones para así potenciar la gobernanza, reducir la pobreza, promover la equidad y potenciar la legitimidad de la acción colectiva.

Desde la perspectiva de la Nueva Gobernanza Democrática (Fleury, 2003), el saneamiento ambiental se define, más allá de lo técnico, como un conjunto de determinantes ambientales de las condiciones de vida a nivel doméstico y comunal, cuya comprensión y abordaje se gestiona en el marco de un red de actores dentro de los que se distingue la representación del Estado y también la presencia activa de la Sociedad Civil (Marsh, 1998) (Klijn, 1998). Es un espacio de construcción de la ciudadanía en el que se hacen valer los derechos a un ambiente sano.

La gestión de las políticas de saneamiento se entiende a partir del concepto de red de políticas públicas, un conjunto de actores interrelacionados que abordan una realidad compleja desde posiciones particulares ajustadas a un conjunto de normas o marco institucional (Luke \& Harris, 2007) (Majone, 1998). Desde esta perspectiva, la unidad de análisis interrelaciones entre actores es fundamental en la gestión (Subirats, 1992), razón que conduce a pensar en un marco institucional amplio e interrelacionado para explicar su comportamiento: un espacio interinstitucional.

En la presente investigación, el análisis de contenido consideró cerca de treinta normas (leyes, reglamentos, normas técnicas) de manera estructurada en cuatro escenarios de gestión. La siguiente figura muestra las principales normas legales correspondientes a cuatro grandes escenarios normativos o instituciones del saneamiento ambiental considerados como más relevantes: el de la atención de la salud en el primer nivel, la gestión del agua para consumo humano, el de gestión de los impactos de la actividad socioeconómica sobre el ambiente y la salud (ordenamiento territorial y la evaluación del impacto ambiental) y el de gestión de los residuos sólidos. 


\section{Figura 1}

Principales escenarios de gestion del saneamiento ambiental en asentamnientos humanos

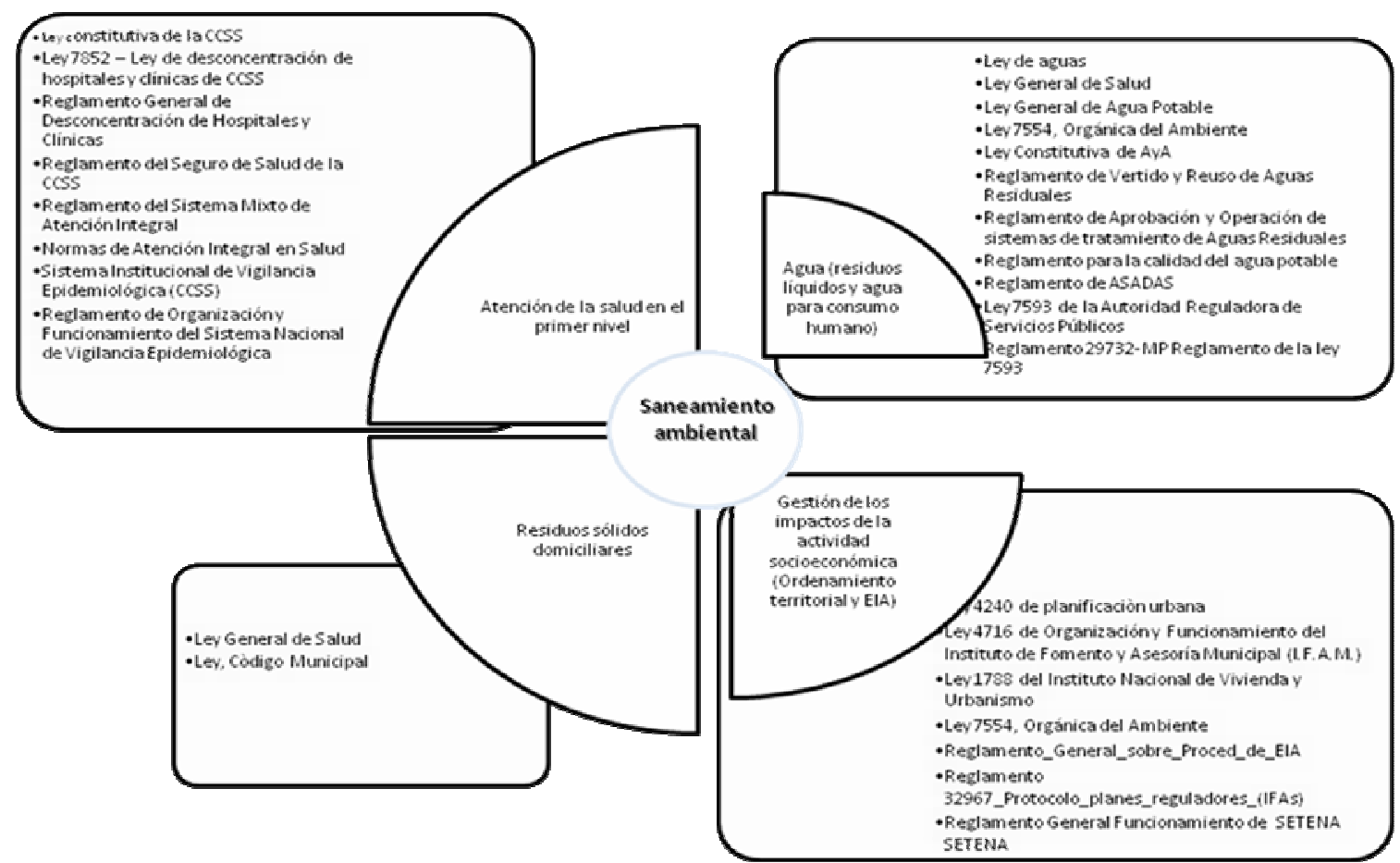

Fuente: elaboración propia a partir de la revisión documental

En la figura anterior se distinguen cuatro grandes escenarios normativos (instituciones formales) que afectan el saneamiento ambiental, unos con más abundante normativa que otros, pero todos en su conjunto deben propiciar un ambiente que promueva la salud y el control de los determinantes ambientales que generan los riesgos y que a su vez conducen a la enfermedad. En síntesis, se orienta a la promoción de la salud, entendida desde la perspectiva ambiental como la búsqueda del bienestar y la mejora de las condiciones de vida.

El análisis de contenido realizado se basa en los principios de la Teoría Fundamentada, es decir, es la realidad de las normas la que ha permitido encontrar puntos de contacto entre los cuatro escenarios e instituciones y ampliar el concepto de saneamiento ambiental. Esto significa la elaboración de un marco comprensivo a partir de la realidad misma (Strauss \& Corbin, 1990).

El análisis se llevó a cabo utilizando el programa Atlas Ti,. Los textos de las normas fueron introducidos en el programa mencionado para ser codificados. Esto permitió caracterizar los conceptos y significaciones sociales implícitos y explícitos en el marco normativo formal, para comprender las particularidades de la gestión del saneamiento ambiental.

El programa Atlas Ti, permite identificar en los textos sometidos al análisis, un sistema de conceptos, significaciones que dan cuenta de las principales ideas o representaciones de la 
realidad que desde el poder político, establecen un orden. El discurso normativo es un principio justificante de la imposición pero no deja de ser un fenómeno ideológico y cultural que refleja y al mismo tiempo promueve una interpretación de la realidad. Es posible identificar entonces una concepción de la realidad que va a ser impuesta en nombre del interés general, por lo que el estudio de los fundamentos conceptuales de la normativa puede ofrecer explicación sobre la organización de los actores en el momento de la implementación de las políticas.

\section{ESCENARIOS INSTITUCIONALES DE GESTIÓN DEL SANEAMIENTO AMBIENTAL EN COSTA RICA.}

La definición de interfase ambiente-salud propone una lectura compleja de los determinantes ambientales del proceso salud-enfermedad, enfoque que se distingue de la perspectiva más tradicional que busca fragmentar los ámbitos de la realidad para propiciar una mejor comprensión a partir de su simplificación (Morin, 1998). Así mismo, se entiende el marco legal del saneamiento ambiental a partir de una red de múltiples normas que deben funcionar articuladamente para asegurar el control efectivo de los riesgos que afectan la salud de las personas y promover la mejora de las condiciones de vida y el desarrollo local.

La articulación de las normas formales se considera un atributo esencial para explicar el comportamiento de los actores que intervienen en la gestión de las políticas públicas. La fragmentación o el aislamiento de escenarios o grandes instituciones, deja espacios vacios que determinan las posibilidades de éxito de la gestión frente a los objetivos de eficiencia, equidad y sostenibilidad.

A continuación se mencionan los principales rasgos encontrados en el análisis de contenido de cada uno de los escenarios de gestión del saneamiento ambiental y finalmente se sintetizan los principales hallazgos. La discusión considera los principales hitos evolutivos de las normas como un elemento que facilita la comprensión de la situación actual, no se pretende así una reconstrucción histórico, sino llamar la atención sobre los principales rasgos presentes en el marco institucional vigente.

\subsection{Escenario de gestión del impacto de la actividad socioeconómica}

Al revisar el grupo de normas legales más relevantes que constituyen el escenario de gestión del impacto de la actividad socioeconómica sobre el ambiente y la salud, sobresalen algunas ideas y conceptos que se van normalizando durante el período de tiempo que se plasman en el siguiente esquema. 


\title{
Figura 2. Evolución del escenario Gestión de impactos de la actividad socioeconómica sobre el ambiente y la salud
}

\author{
Evolución del escenario \\ Gestión de los impactos de la actividad socioeconómica sobre \\ el ambiente y la salud
}

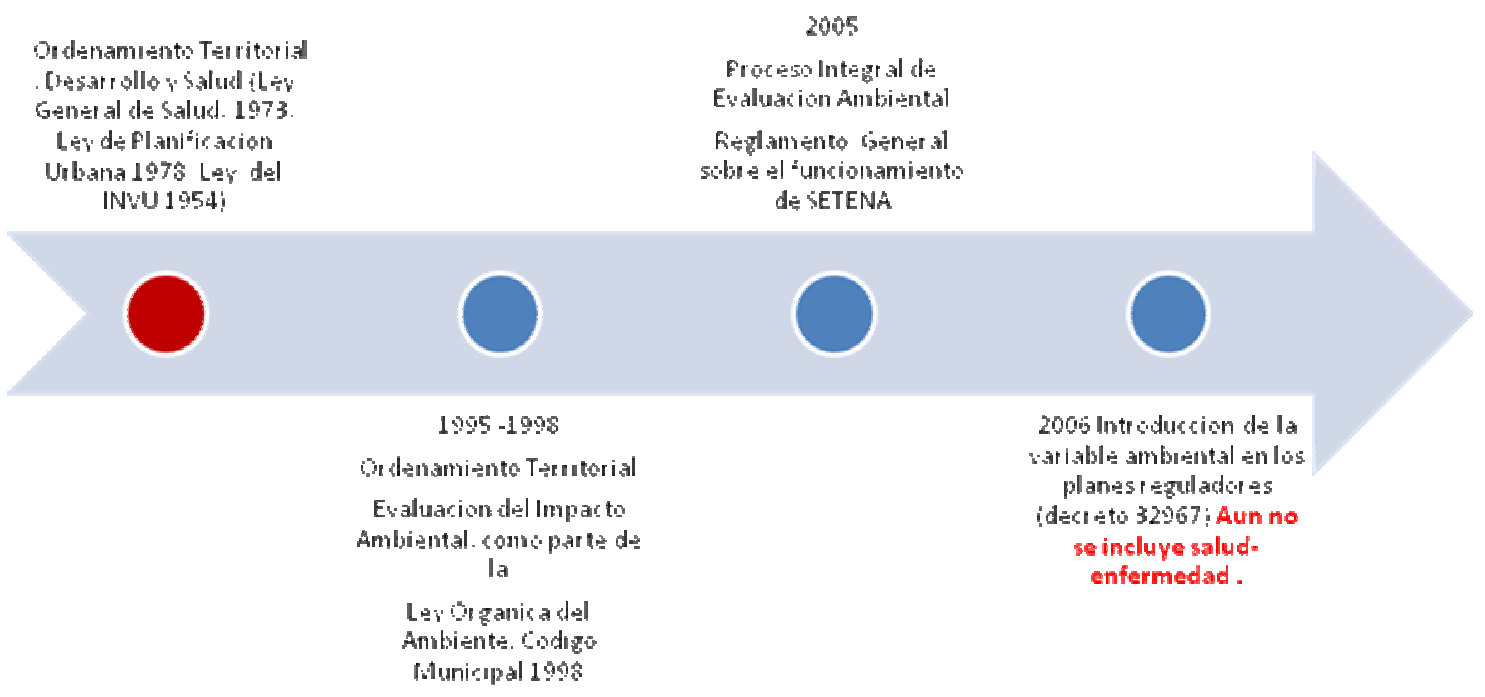

Fuente: elaboración propia a partir de la consulta de leyes y reglamentos de la república.

En el esquema anterior se distinguen algunas ideas y conceptos relevantes que se transforman en normas legales para regular el espacio geográfico del asentamiento y como una parte muy importante del mismo, el saneamiento ambiental:

- la idea de que el espacio urbano e implícitamente el de asentamientos humanos, se debe planificar. La apropiación del espacio urbano no es un proceso racional y espontáneo

- Es un proceso que se debe hacer localmente para reconocer las necesidades sentidas de los habitantes y

- debe incidir directamente en el desarrollo y la salud de los habitantes.

Estas ideas y conceptos se formulan como leyes de la república entre los años 1950 y 1970 quedando plasmadas como tal con vigencia hasta el actual período de análisis. Sin embargo, aunque se trata de ideas y conceptos vigentes desde hace varias décadas, toman forma legal más precisa y se interrelacionan a partir de 1995 cuando se enuncia y establece la Ley Orgánica del Ambiente.

A partir de 1995-1996 se comienzan a desarrollar los Planes Reguladores en Costa Rica, sin embargo, no es hasta la presente década y hacia el final del período de estudio (año 2008) que se operacionaliza de manera efectiva mediante los reglamentos de la Secretaria Técnica Ambiental (SETENA), la importancia de que estas políticas de desarrollo local tengan un sustento 
ambiental. En el momento de cierre de la presente investigación aun no se había concedido la Licencia Ambiental a ningún plan regulador en curso (MINAE, 2006), lo que evidencia que se trata de una idea fructífera, pero aun en desarrollo.

Como se ha explicado, la integración de la variable ambiental aun no ocurre como proceso finalizado en ninguno de los planes reguladores definidos. Pero aun más controversial resulta que la inclusión de la variable salud-enfermedad como proceso ligado al ambiente y al desarrollo, sigue sin estar clara (a nivel operativo) y no aparece incluida explícitamente en la normativa legal vigente en Costa Rica.

Es de destacar además que el modelo de gestión de políticas para regular este escenario se concibió como un proceso exclusivamente técnico. Incluso, en la Ley Orgánica del Ambiente (Asamblea Legislativa de la República de Costa Rica, 1995) de 1995 existe un capítulo (el capítulo 2) sobre participación ciudadana, pero este se reduce a discutir las políticas establecidas desde "arriba" y hacer propuestas de proyectos y actividades que no necesariamente se terminan llevando a cabo porque no son vinculantes.

La perspectiva de interacción entre actores como estrategia para solucionar conflictos locales ligados al desarrollo, se operacionaliza un poco más claramente a partir de 1995 como consulta ante las propuestas técnicas. Esto es algo accesorio a los procesos de toma de decisión científicotécnico. Actualmente, como se ha explicado, no ha sufrido grandes variaciones.

Después de revisar este escenario institucional se distinguen algunos rasgos que pueden comprometer el control de las interacciones ambiente-salud y terminar afectando la satisfacción de las necesidades de saneamiento ambiental en los asentamientos humanos:

- El escaso vínculo entre el ordenamiento territorial como actividad fundamentalmente correctiva de los impactos, con la Evaluación del Impacto Ambiental como actividad eminentemente preventiva. El vínculo entre prevención y corrección se logra formalizar legalmente al final del período de estudio, pero demanda un alto grado de información y conocimiento a todo nivel que puede comprometer su efectividad.

- Desde el punto de vista conceptual, ambos subestiman y no operacionalizan los impactos ambientales en la salud, lo que se evidencia al limitar las membrecías en la redes de actores, particularmente para los actores relacionados directamente con la atención de la salud.

- No se asume el tema de la educación como una función que puede ayudar no solo a la mejor comprensión del impacto y los riesgos, sino estimular una participación directa de la ciudadanía. Así mismo, la normativa no presta atención a la construcción colectiva de la información y el conocimiento como una vía para propiciar el debate y el reconocimiento de la diversidad de intereses.

- El limitado ejercicio de ciudadanía participativa, lo que puede comprometer la representación de la diversidad de intereses en la gestión del saneamiento ambiental y particularmente el interés de los afectados directos por los efectos adversos del crecimiento socioeconómico.

- Se reduce a núcleos urbanos, dejando a los asentamientos rurales fuera de la gestión de los impactos. 


\subsection{Escenario de gestión del agua para consumo humano}

Al revisar la evolución de las ideas y conceptos más relevantes sobre la gestión del agua para consumo humano se destacan los principales hitos en el siguiente esquema:

Figura 3. Evolución del escenario de gestión del agua para consumo humano

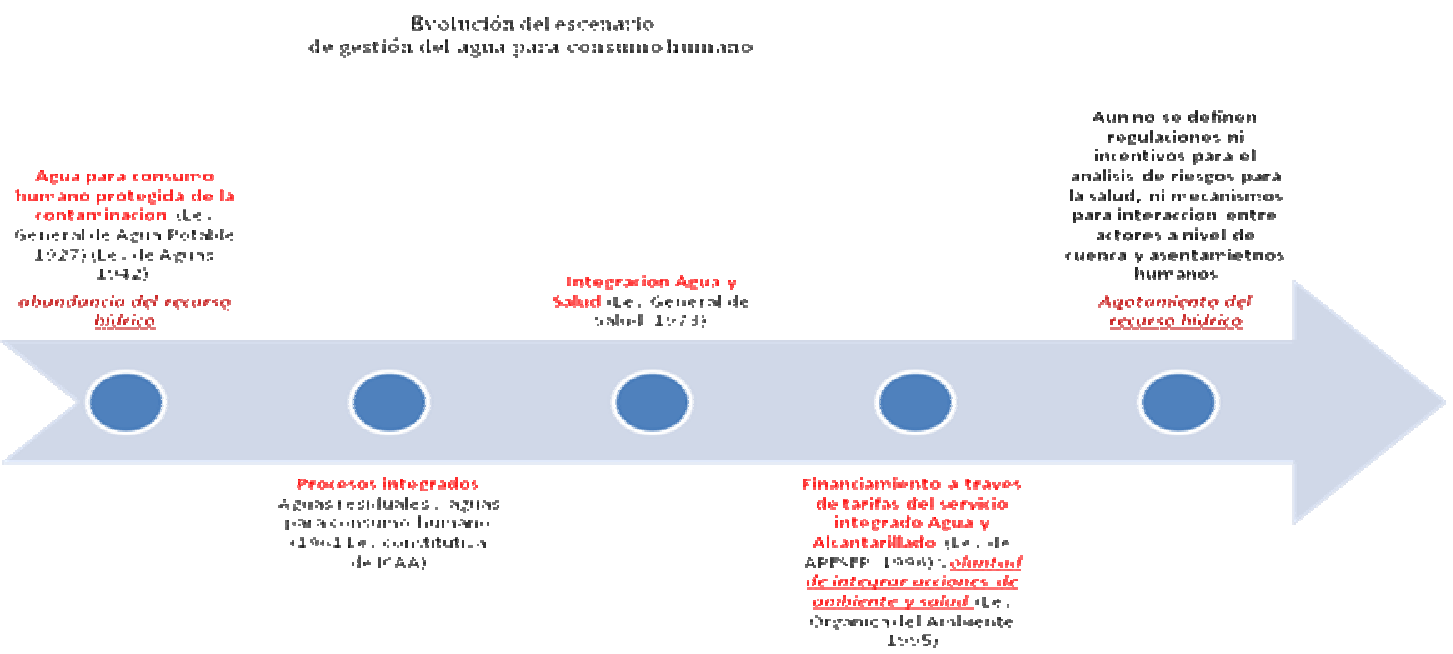

Fuente: Fuente: elaboración propia a partir de leyes y reglamentos

En el esquema anterior se muestra que las ideas en torno a la protección del agua para consumo humano frente a las amenazas de la contaminación ya están plasmadas en las normativas legales de la época (entre 1927 y 1942), probablemente porque ya se entendían los vínculos con la salud de las personas a pesar de que esto se produce en condiciones de relativa abundancia del recurso hídrico. A partir de este es momento va cobrando fuerza el interés por responsabilizar al Estado del agua para consumo humano, responsabilidad que se extiende hacia el alcantarillado sanitario.

Sobre la constitución del Instituto Costarricense de Acueducto y Alcantarillados (ICAA) en 1961, es importante tomar en cuenta que se vinculan bajo una misma organización el agua para consumo humano y el manejo de los residuos líquidos, buscando una gestión integrada. Sin embargo, probablemente la idea en aquel momento sobre la gestión de residuos líquidos se reducía a alcantarillado, concepción que continua predominando en la actualidad y que como se ve constituye a la propia organización del ICAA.

La reducción conceptual que se hace del manejo de residuos líquidos a alcantarillado significa por una parte, el predominio de la idea de abundancia del recurso hídrico (bajo estas condiciones funciona el alcantarillado) y por otro la necesidad sentida de darle solución a las amenazas de contaminación del agua y controlar los riesgos para la salud humana. 
La Ley General de Salud de 1973, expresa una consolidación de las ideas que se tienen sobre los vínculos entre agua para consumo humano y salud. Se muestra una noción muy clara sobre los riesgos para la salud asociados al agua, lo que debe contribuir a reforzar la idea del agua como servicio público con el que el Estado debe tener un compromiso sólido. A través del Ley Orgánica del Ambienta se plantea con claridad la voluntad de integrar acciones ambientales y las de salud, y se crean los consejos ambientales regionales y el nacional con participación intersectorial.

Posteriormente, a estas ideas se asocia la perspectiva económica del recurso. El escenario no se puede gestionar al margen de sus costos, por eso se ha definido una autoridad reguladora, para asegurar así el financiamiento y la calidad del servicio. Sin embargo, esta idea que se estableció como norma legal, simplemente se adicionó a las concepciones sobre recurso hídrico-agua para consumo humano-riesgos para la salud, sin generar grandes transformaciones. Esto es porque el análisis de costos que supone, se reduce al servicio de acueducto estrictamente y no es suficientemente explícita sobre los costos de la protección del recurso (costos ambientales) y las amenazas para la salud.

Se identifica a lo largo de línea de tiempo que el problema del agua (residuos líquidos y agua para consumo humano), se ha visto sobre todo como un problema científico-técnico que el Estado ha ido asumiendo de manera progresiva, cada vez con mayor compromiso por regular este ámbito estrechamente ligado a la salud. Pero no se ve como un problema sociopolítico que se deba resolver a partir de las interacciones entre actores, utilizando mecanismos que permitan superar los conflictos particulares que se presentan en cada sistema de abastecimiento de agua y asentamientos.

A pesar de que el contexto de relativa abundancia del recurso ha ido cambiando de manera gradual y al final de esta línea del tiempo se reglamenta de manera estricta los vertidos de contaminantes al agua y se promueven acciones para la inspección, vigilancia y control de la calidad del agua para consumo humano, aun se aprecian numerosos aspectos deficitarios que se han discutido en este epígrafe. Desde el punto de vista técnico, se nota la falta de vínculo entre recurso hídrico- agua-riesgos para la salud; conjunto de conceptos que aun se manejan desintegradamente.

Desde el punto de vista sociopolítico, no se evidencia una evolución el modelo establecido de gestión burocrática a través de un solo actor que es la organización del Estado competente técnicamente en este tema. Esto podría obstaculizar el abordaje instersectorial del problema del agua y la promoción de una ciudadanía activa desde este punto de vista. Así mismo, se podría comprometer la gestión eficiente, equitativa y sostenible de los problemas y necesidades en esta materia.

\subsection{Escenario de gestión de residuos sólidos}

El escenario de gestión de residuos sólidos en asentamientos humanos es probablemente el menos desarrollado a nivel de normas legales. No existe en el momento de cierre de la presente investigación, una ley o reglamento específico que regule o establezca servicios orientados hacia los residuos sólidos en asentamiento humanos (se discute un proyecto de ley en la Asamblea Legislativa). En el siguiente esquema se plasman las principales ideas al respecto: 


\section{Figura 4. Evolución del escenario de gestión de residuos sólidos}

Eroluctón dal ascenat:

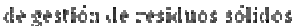

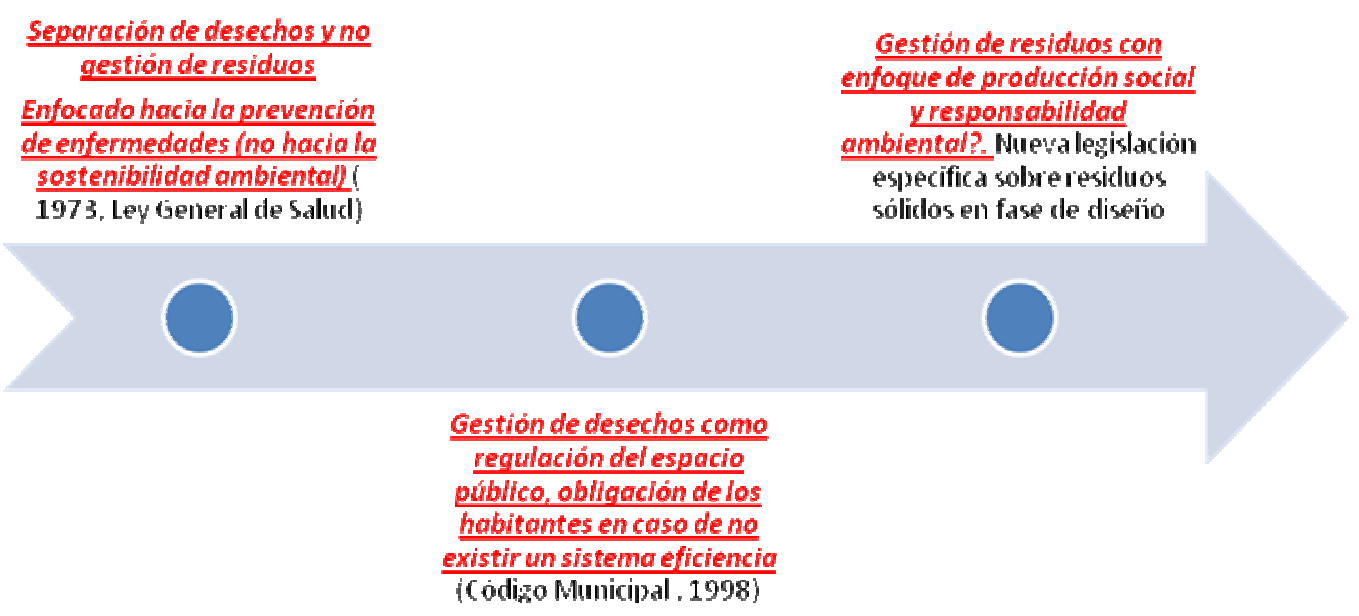

Fuente: Fuente: elaboración propia a partir de la revisión de normas legales

Como se ve en el esquema anterior, desde la perspectiva sanitaria, a través de la Ley General de Salud, el enfoque está sesgado hacia la prevención de enfermedades. Los residuos no se dejan de ver como desechos en el Código Municipal, un proceso que debe ser regulado por el Ministerio de Salud. Se continúa con la perspectiva de solucionar el problema de la "basura" apartándola, los habitantes juegan un papel secundario pues estarían obligados a participar en el caso de que la municipalidad no logre resolver el problema.

No se aprecia aun una evolución de la idea y los conceptos que permita posicionar el enfoque de gestión de residuos, con perspectiva de sostenibilidad ambiental y que considere el problema de consumo como un problema de producción de residuos del cual los habitantes y los productores tienen responsabilidad.

Un rasgo importante es la desconcentración de la gestión ahora en manos de la municipalidad, y el hecho de que de alguna forma se vea como un problema sanitario. Sin embargo, se corre el riesgo de que con esto se diluya la responsabilidad del gobierno central y se obstaculice la lectura del problema y las soluciones como un problema de Estado y de toda la sociedad.

El cambio que implica el enfoque de gestión de residuos sólidos se orienta más que a la prevención de la enfermedad hacia la producción social de la salud. Se supone así que este no es solo un problema técnico que se soluciona con la separación a nivel doméstico y una buena disposición, sino que es un problema social y político. Debe ser un proceso que vincule a productores y consumidores preocupados y participando por la sostenibilidad ambiental y la promoción de la salud. 


\subsection{Escenario de atención de la salud en el primer nivel}

Al revisar los principales hitos evolutivos que definen la atención primaria en el primer nivel de atención desde el punto de vista de la atención de los determinantes ambientales en asentamientos humanos es de destacar los siguientes rasgos:

- Los principios de universalidad (la cobertura de todos los habitantes con servicios de atención a las personas y al ambiente), la solidaridad y la equidad (reducir las inequidades entre grupos poblacionales). Estos principios están vigentes desde la constitución de la Caja Costarricense del Seguro Social (CCSS) y se reafirman con la extensión del régimen del seguro social en 1971 y 1973 cuando se extienden los regímenes de la CCSS y son trasladados los servicios de atención médica del Ministerio de Salud a la CCSS)

- La separación de la atención curativa y la rehabilitación (en manos de la CCSS) de los servicios de prevención de enfermedades y promoción de la salud (en manos del Ministerio de Salud), todo esto a partir de 1973.

- La readecuación del modelo casi veinte años después, hacia la atención integral, momento en el que se trasladan los servicios de prevención de enfermedades y promoción de la salud del Ministerio de Salud a la CCSS. El Ministerio de Salud comienza un proceso de cambio hacia la rectoría, se define el sistema nacional de salud con un primer nivel que enfatiza en prevención y en promoción y se articula con el resto de los servicios. (Ministerio de Salud, 1992)

- La expresión práctica de una voluntad eficientista que se basa en la creación de condiciones de cuasimercado: desconcentración, separación de las funciones de financiamiento, compra y prestación de servicios, participación de la Sociedad Civil como mecanismo de fiscalización de la eficiencia y la calidad.

Esta sucesión de acontecimientos se esquematiza a continuación:

Figura 5. Evolución del escenario de la atención de la salud en el primer nivel

Evouluciōn del escestay

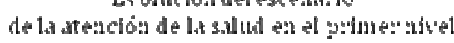

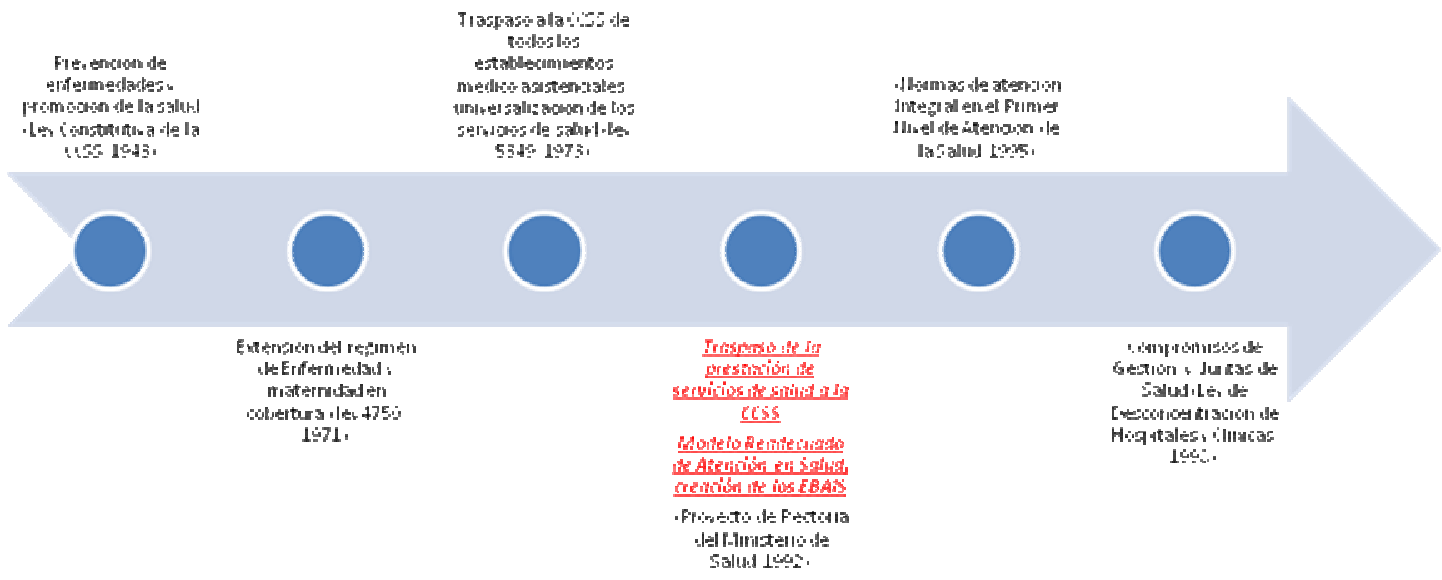

Fuente: elaboración propia a partir de la revisión de leyes y reglamentos 
Las transformaciones están marcadas por cambios en las normas legales que pueden influir sobre la atención del ambiente desde la perspectiva de sanitaria de la siguiente forma:

- La tendencia a la universalidad, la solidaridad y la equidad favorece sin dudas la atención de los determinantes sociales de la salud más allá de los individuales. Esto pudo haber favorecido al desarrollo de una visión colectiva de la salud y al desarrollo de estrategias de atención del ambiente desde el sector sanitario. Un ejemplo de esto son los programas de saneamiento ambiental como Saneamiento Ambiental Rural (SANEBAR) aun vigente.

- La separación de funciones entre la CCSS con lo curativo y el Ministerio de Salud en lo preventivo, durante cerca de veinte años, pueden contribuir a que la CCSS desarrollara más un vocación curativa orientada hacia la biología y la enfermedad y el Ministerio de Salud lo hiciera hacia la prevención y la promoción con perspectiva más social hacia la salud.

- En el momento que se readecua el modelo de atención: se define el primer nivel, pasa a manos de la CCSS la prevención y la promoción y se estructura el sistema de atención; probablemente no hay condiciones creadas para que estas políticas alcancen un buen nivel de implementación: la CCSS no tiene experiencia ni un cultura para llevar a cabo esto, el rector Ministerio de Salud se encuentra debilitado con limitada capacidad (que ha sido transferida a la CCSS, no cuenta con recursos legales ni técnicos para hacer rectoría) y carece de una cultura para el ejercicio de la regulación. El resultado es que las acciones de prevención y promoción pueden caer en un "vacío de implementación".

- A pesar de la experiencia en prevención y en promoción de la salud, las normas de atención integral definidas por el Ministerio de Salud, se orientan más a la enfermedad y lo curativo. Situación que no ayuda hacia una proyección de la CCSS hacia la atención del ambiente.

- La aplicación de principios de eficiencia en la prestación del servicio a partir de 1998, podría contribuir a reproducir y reafirmar la cultura de la CCSS en lo biológico-curativo, dejando un déficits de trabajo en el tema ambiental y particularmente en lo que tiene que ver con saneamiento ambiental.

- Como se ve, la consecución de hechos relacionados con la transformación del marco normativo legal de los servicios de salud en el primer nivel, pueden conducir a una desatención de los determinantes ambientales de la salud desde la perspectiva de los servicios sanitarios. Así mismo, cabría la interrogante sobre cuál es el punto de partida de la concepción de la promoción de la salud y como parte de esta la Salud Ambiental, en el marco del modelo de atención.

\section{LA GESTIÓN DEL SANEAMIENTO AMBIENTAL Y LA PRODUCCIÓN DEL SANEAMIENTO AMBIENTAL EN COSTA RICA.}

La gestión del saneamiento ambiental desde una perspectiva compleja se entiende como la conjunción de escenarios institucionales. Así mismo, el marco normativo formal que lo enmarca se estructura al menos en cuatro escenarios (instituciones): el de la atención de la salud en el primer nivel, la gestión del agua para consumo humano, el de gestión de los impactos de la actividad socioeconómica sobre el ambiente y la salud (ordenamiento territorial y la evaluación del impacto ambiental) y el de gestión de los residuos sólidos. Sin embargo, los escasos puntos de contacto que se han constatado durante el análisis de contenido pueden dificultar su gestión integrada, un requisito que define su propia existencia.

La evolución en el tiempo del escenario de gestión del impacto de la actividad socioeconómica en el ambiente y la salud ha estado marcada por una tendencia a la integración de instrumentos normativos que promueven el desarrollo con sostenibilidad ambiental. Sin embargo, otros 
escenarios institucionales como el de la gestión del agua para consumo humano, aun está lejos de considerar como parte del mismo proceso de gestión la disposición de residuos líquidos.

Por otra parte, el marco normativo de la gestión de residuos sólidos es probablemente el menos evolucionado de todos pues no cuenta con una ley específica y se conciben los residuos como basura, lo que evidencia la escasa conciencia sobre su valor económico y ambiental. Hacia el cierre la presente investigación, se encuentra en fase de diseño una ley específica sobre el tema que podría favorecer una mejor gestión de los residuos sólidos en el marco de los asentamientos humanos.

El escenario del primer nivel de atención de la salud, es uno de los que más cambios ha presentado. Las leyes que lo sustentan constituyen una estructura favorable para la gestión del saneamiento ambiental desde la perspectiva de salud, pero las normas técnicas y reglamentos aun no consideran de manera suficiente esta posibilidad pues deben enfrentar el paradigma biomédico reproducido y afianzado durante mucho tiempo.

Al revisar las normas formales que constituyen los escenarios de gestión se identifican hitos que han puesto en evidencia la tendencia a definir puntos de contacto entre el ambiente, el desarrollo y la salud. Sin embargo, aun no se visualiza un nivel de integración aceptable que viabilice la conformación de constelaciones de actores amplias e integradas así como modos de relación negociados entre actores que permitan la atención de la complejidad de las relaciones ambientesalud y particularmente el saneamiento ambiental. La situación, más allá de la voluntad de cada actor, no propicia la construcción colectiva del conocimiento sobre la interfase ambient-salud y menos aun su gestión integrada.

Los incentivos que se identifican en las normas legales favorecen la adopción de soluciones ágiles definidas predominantemente con criterio científico-técnico, las que no favorecen la confrontación de opiniones y la incorporación de intereses diversos. De manera que la información concebida durante la puesta en práctica es eminentemente técnica y el conocimiento se construye entre muy pocos actores debido a que no se definen relaciones negociadas o de coordinación con actores de la Sociedad Civil en los espacios geográfico-poblacionales. Las limitaciones en la construcción social del conocimiento evidencian dificultades claras para asegurar la producción social de la salud.

Al revisar los roles asignados a los actores sobresale el de la rectoría que se materializa en la autoridad sanitaria: El Ministerio de Salud. El rector de la salud tiene una escasa presencia a nivel de asentamientos humanos en diversos escenarios como es de la gestión del impacto de la actividad socioeconómica sobre el ambiente y la salud, así como el de la gestión del agua para consumo humano. Esto puede comprometer el ejercicio del poder y la representación del Estado en esta arena de políticas públicas.

Se observó, al revisar el marco normativo formal que predominantemente las relaciones entre el rector de la salud y los entes regulados se producen a partir de acciones unilaterales o negociaciones que en notable medida dejan por fuera al resto de la Sociedad Civil, potencialmente afectada por las decisiones tomadas y por sus consecuencias. Esto significa que la Sociedad Civil no tiene participación directa en todo el proceso de gestión que resulta dominado fundamentalmente por criterios técnicos. 
El carácter limitado o incompleto de las redes definidas legalmente puede incidir en que no existan vínculos explícitos entre la atención del ambiente y la atención de la salud en el marco territorial de los asentamientos humanos y además la concentración del poder en organizaciones del Estado que hacen valer sus capacidades técnicas..

En síntesis, a pesar de la voluntad de los actores involucrados y la elocuencia del discurso con enfoque social, la lógica de lo que es adecuado hacer en materia de saneamiento ambiental en asentamientos humanos aun está distante de promover la gestión integrada del saneamiento ambiental. Las reglas del juego vigente no favorecen la construcción de una ciudadanía activa, pero sobre todo la prevención de enfermedades y la promoción de la salud desde la perspectiva de la producción social.

\section{REFERENCIAS BIBLIOGRÁFICAS}

Asamblea Legislativa de la República de Costa Rica. (setiembre de 1995). Ley Orgánica del Ambiente. Recuperado el 13 de febrero de 2008, de www.asamblea.go.cr

Fleury, S. (2003). Reforma del Estado. Revista Instituciones y Desarrollo , 81-122.

Giandoménico, M. (1998). Public Policy and Administration: ideas, interest and institutions. En E. Goodin, \& H. D. Klingemann, A new handbook of Political Science (págs. 610-628). New York: Oxford University Press.

Insituto Federal Suizo de Ciencia y Tecnología Acuática. (2006). Saneamiento Ambiental Centrado en el Hogar (HCES). Ginebra: EAWAG.

Klijn, E. (1998). Policy Networks. En W. Kickert, \& J. F. Koppenjan, Managing Complex Networks. London: Sage.

Luke, D. A., \& Harris, J. (2007). Network analysis in Public Health: history, methods and applications. Annu Rev Public Health (28), 69-93.

Majone, G. (1998). Public Policy and Administration: ideas, interests and institutions. En R. E. Goodin, \& H. D. Klingemann, A New Hanbook of Political Science (págs. 610-628). New York: Oxford.

Marsh, D. (1998). Comparing policy networks. Public policy and management. Buckingham Philadelphia: Open University Press.

MINAE. (julio de 2006). MINAE. San José, Costa Rica.

Ministerio de Salud. (1992). Proyecto de rectoría y fortalecimiento del Ministerio de Salud. San José: Unidad de Preparatorio del Proyecto. 
Morin, E. (1998). Introducción al pensamiento complejo. Barcelona: Gedisa Editorial.

North, D. (1993). Instituciones, cambio social y desempeño económico. México: Fondo de Cultura Económica.

OMS, Comission on Social Determinans of Health. (2007). Civil Society Report. Ginebra: OMS.

Peters, G. (2003). El nuevo institucionalismo. Barcelona: Ciencia Política.

Rangeon, F. (1986). La ideología del interés general. Paris: Editorial Económica.

Rhodes, R. (1997). El "institucionalismo". En D. Marsh, \& G. Stoker, Teoría y Métodos de la Ciencia Política (págs. 53-67). Madrid: Alianza.

Rivas Leone, J. (2003). El neoinstitucionalismo y la revalorización de las instituciones. Mexico: Panorama.

Sojo, A. (2000). Reformas de gestión en salud en América Latina: los cuasimercados de Colombia, Argentina, Chile y Costa Rica. Santiago de Chile: CEPAL.

Strauss, A., \& Corbin, J. (1990). Basics of qualitative research: Grounded Theory procedures and techniques. Newbury Park-London-New Delhi: Sage.

Subirats, J. (1992). Análisis de políticas públicas y eficacia de la administración. Madrid: Ministerio para las Administraciones Públicas. 


\section{AGRADECIMIENTOS}

El autor reconoce el apoyo del Swiss National Centre of Competence in Research (NCCR) North-South: Research Partnerships for Mitigating Syndromes of Global Change, co-financiado por Swiss National Science Foundation (SNF) y la Swiss Agency for Development and Cooperation (SDC).

El autor reconoce el apoyo del Doctorado en Gobierno y Políticas Públicas de la Universidad de Costa Rica por el soporte y asesoría para el desarrollo de la investigación 